\title{
Fecal microbiota transplant - a new frontier in inflammatory bowel disease
}

This article was published in the following Dove Press journal: Journal of Inflammation Research

\section{Tagore Sunkara' \\ Prashanth Rawla ${ }^{2}$ \\ Andrew Ofosu' \\ Vinaya Gaduputi ${ }^{3}$}

'Division of Gastroenterology and Hepatology, The Brooklyn Hospital Center, Mount Sinai Health System, New York, NY, USA; ${ }^{2}$ Department of Internal Medicine, Sovah Health, Martinsville, VA, USA; ${ }^{3}$ Division of Gastroenterology, SBH Health System, Bronx, NY, USA
Correspondence: Prashanth Rawla Department of Internal Medicine, Sovah Health, 320 Hospital Drive, Martinsville, VA 24II5, USA

Tel +l 8602184942

Email rawlap@gmail.com

\begin{abstract}
Inflammatory bowel disease (IBD) is a chronic multifactorial disease that affects the gastrointestinal tract and results from an aberrant immune response toward luminal antigens in genetically susceptible people. Most of the current therapies for IBD focus on the management of the inflammation by using corticosteroids, immune modulators, and more recently, monoclonal antibodies (biological therapy). Although these therapies provide benefit in most cases, there are still a significant number of patients who do not respond or become refractory over time, suggesting the need for alternative therapeutic options. In the last decade, it has been recognized that "dysbiosis," an imbalanced gut microbiota, is a key element in IBD suggesting microbiome-based therapies as an attractive approach. Recently, fecal microbiota transplant (FMT) has been successfully used for the treatment of Clostridium difficile infection, and it is now under investigation for the treatment of IBD. Clinical trials data are still poor but strongly support a future introduction of FMT in therapy to manage IBD microbiome. More studies are needed to assess the optimal route of administration and the frequency of FMT, the best matched donor for each patient as well as the risks associated with FMT in IBD.
\end{abstract}

Keywords: fecal microbiota transplant, FMT, inflammatory bowel disease, IBD, microbiome, dysbiosis, ulcerative colitis, Crohn's disease

\section{Introduction}

\section{Pathophysiology of inflammatory bowel disease (IBD) and the use of fecal microbiota transplant (FMT)}

Inflammatory bowel disease is a common gastrointestinal (GI) disorder affecting approximately $10 \%-15 \%$ of the worldwide population, ${ }^{1}$ characterized by chronic abdominal pain and altered bowel habits. Although the pathophysiology of IBD remains still largely unknown, growing evidence suggests that factors such as food habits, antibiotics, and gender, as well as psychosocial events, ${ }^{2}$ may be a trigger in genetically susceptible individuals. ${ }^{3,4}$ In the recent years, an important role of the gut microbiota in IBD has been recognized. In the healthy status, the gut microbiota actively interacts with the human host to modulate several physiological functions such as gut development, ${ }^{5}$ immune responses, ${ }^{6,7}$ resistance to pathogens, ${ }^{8}$ and brain development and functions. ${ }^{910}$ Changes in bacteria composition and number, known as "dysbiosis," have repeatedly been observed in IBD patients and are now recognized as a key element in gut inflammatory processes. ${ }^{11-13}$ Dysbiosis of the gut microbiota is characterized by a significant reduction of obligate anaerobes such as members of the phyla Bacteroidetes and Firmicutes and a sharp increase in facultative anaerobes 
such as the phyla Actinobacteria and Proteobacteria, such as Escherichia coli. A decrease in obligate anaerobes results in the release of anti-inflammatory compounds, which causes increased inflammation. ${ }^{14,15}$ Therefore, the dysbiosis correction has been considered an attractive therapeutic approach. FMT can reduce bowel permeability by increasing the production of short-chain fatty acids, especially butyrate, which helps in maintaining the integrity of the epithelial barrier and thus decreases the severity of the disease. FMT can also restore immune dysbiosis by regulating the inflammatory markers by inhibiting the activity of $\mathrm{T}$ cells, leukocyte adhesion, and the production of inflammatory factors. ${ }^{16}$ Although microbiome-based therapies, such as probiotics and antibiotics, failed to be effective in IBD, recently, FMT is gaining a new life as a therapeutic option for the patients with a disturbed gut ecosystem.

FMT is a procedure in which fecal or stool matter is collected from a healthy donor and placed into a patients' GI tract to correct the dysbiosis and to restore healthy conditions. ${ }^{17}$ FMT has already been successfully used for the treatment of recurrent Clostridium difficile infection (rCDI) resistant to conventional antibiotic therapies, ${ }^{18,19}$ with an efficacy $>90 \% .{ }^{20-23}$ For this reason, it is now being considered as an experimental therapy in IBD and labeled by the Food and Drug Administration as Investigational New Drug in 2016. ${ }^{24}$

\section{Preparation and routes of administration of the new microbiota}

There has been an increasing interest in the use of FMT for chronic GI infections and IBDs. ${ }^{25}$ FMT requires few important steps prior to transplant that are: 1) preparation of the fecal material and 2) selection of the route of administration.

\section{Preparation of FMT}

Prior to FMT, the stool is collected by the donor using a sterile collection kit provided in advance. The stool can be used either immediately or frozen for later use. According to many protocols, at least 30-50 g of freshly produced stool is sufficient for a successful FMT. ${ }^{26,27}$ However, the stool's weight is not an accurate measure of the microbiota quantity as it may vary among donors. Once collected, the stool is diluted in a volume of a sterile saline from three to five times larger than the starting fecal material (ie, $30 \mathrm{~g}$ of stool is mixed with $150 \mathrm{~mL}$ of saline). In addition, other solvents have been used such as water, yogurt, and milk. ${ }^{28,29}$ However, whether saline is preferable to preserve the microbes ${ }^{30}$ better is still unknown because of the lack of comparative studies. Following homogenization, the preparation is filtered (using, eg, gauze, coffee filter, and strainer), and particles $<2 \mathrm{~mm}$ in width are allowed to prevent any clog of the infusion tube during colonoscopy. ${ }^{31} \mathrm{Cui}$ et al reported about automatic purification of microbiota from stool using microfiltration and centrifugation. ${ }^{32}$ The solution is then directly infused in the GI tract or further centrifuged to obtain a pellet composed by bacteria to be placed in gelatin capsules for oral administration. To do this, the pellet is then suspended to obtain a working concentration of $0.5 \mathrm{~g} / \mathrm{mL}$ and rapidly frozen in 5 - or $10 \mathrm{~mL}$ volume syringes. Size 1 acid-resistant hypromellose capsules are filled with $0.4 \mathrm{~mL}$ of fecal material and placed in size 0 acid-resistant hypromellose capsule and then nested within size 00 gelatin capsule. ${ }^{33}$ Freshly prepared stool must be delivered preferably within 6 hours after emission; otherwise, the preparation must be discarded. The use of fresh material may have some logistical limitations, such as the cost of the technique, the donors' availability, and the time associated with the screening. Recently, FMT approach using frozen materials has been developed and filled those gaps. ${ }^{34}$ Indeed, the frozen material offers a larger pool of stool samples to choose from allowing the availability of stool on demand as well as the selection of the best match between donors and recipients. Similar to fresh samples, the preparation of frozen stool consists of dilution and homogenization of the sample. Before freezing, glycerol to a $10 \%$ final concentration should be added ${ }^{29}$ to protect microbial cells from the damage induced by freezing. ${ }^{35}$ Although the optimization of the storage temperature is still under debate, it is preferable at $-80^{\circ} \mathrm{C}$, because some enzymes are still active at $-20^{\circ} \mathrm{C}$. ${ }^{36}$

\section{Route of administration}

Different ways to deliver the new microbiota have been reported via the lower GI tract or upper GI tract.

By the lower digestive tract, FMT is given by colonoscopy or rectal enema. After appropriate anesthesia, an amount of $200-500 \mathrm{~mL}$ of donor stool is infused to the colon (terminal ileum, cecum, or sigmoid) via the endoscope channel. ${ }^{21,28,37}$ FMT performed via colonoscopy carries the risk associated with colonoscopies, such as adverse sedative reaction and bowel perforation. Patients who are not suitable for colonoscopy may receive FMT via enema or the upper GI tract. When applied through the upper GI tract, the total volume of donor stool suspension is 10 times lower $(25-50 \mathrm{~mL})$, and it is delivered through nasogastric, nasojejunal, or gastrostomy tube, keeping the patient at $45^{\circ}$ upright position for 4 hours after infusion to prevent aspiration. ${ }^{38}$ However, this is the method that patients prefer less. Recently, gelatin-coated or frozen capsules are under investigation to increase the avail- 
ability of FMT therapy in terms of accessibility and patient compliance. ${ }^{33,39}$ Upon filling and capping, 8-12 capsules are administered to patients. ${ }^{39}$ FMT is reported to be effective by all of the routes, and the preferred method may vary with the clinical situation. ${ }^{17}$ Less invasive methods, such as retention enema and nasointestinal infusion, may be safer in patients who are frail or severely ill at the time of FMT. Transendoscopic enteral tubing for FMT through the colon has been reported by Peng et al. ${ }^{40}$ Other methods of delivery through small intestine stoma or percutaneous endoscopic gastrojejunostomy have also been reported. ${ }^{40,41}$

The choice of the delivery route will depend on the type of microbiota to transplant (gastric juice can activate certain bacteria species and damage others), the overall health status of the patient, and the disease to be treated (CDI vs colitis). In addition, the number of administration is crucial, because a single dose may be adequate for CDI, but not for IBD. Furthermore, despite the beneficial effect of the transplanted microbiota, it is known that the host shapes the microbiota, ${ }^{42,43}$ suggesting that host genes and diet can limit FMT effectiveness. Figure 1 summarizes various routes of administration of FMT.

\section{Donor selection, screening, and stool composition to impact the efficacy of FMT}

Donor selection is a big challenge for FMT in IBD. Because the major concern of the FMT is the possibility to spread viral or bacterial infections through the stools, FMT requires a healthy donor chosen among patient-selected (relative or friend) or anonymous person with no risk factors for transmissible diseases or any issues that may alter the microbiota composition, such as drugs or antibiotics. Thus, potential donors undergo a selection process that involves a preliminary interview followed by at least two rounds of blood and stool tests. ${ }^{44}$

In the first step, all potential donors fill out a questionnaire about their medical history and life habits to identify risk factors that can be eventually undetectable through the blood

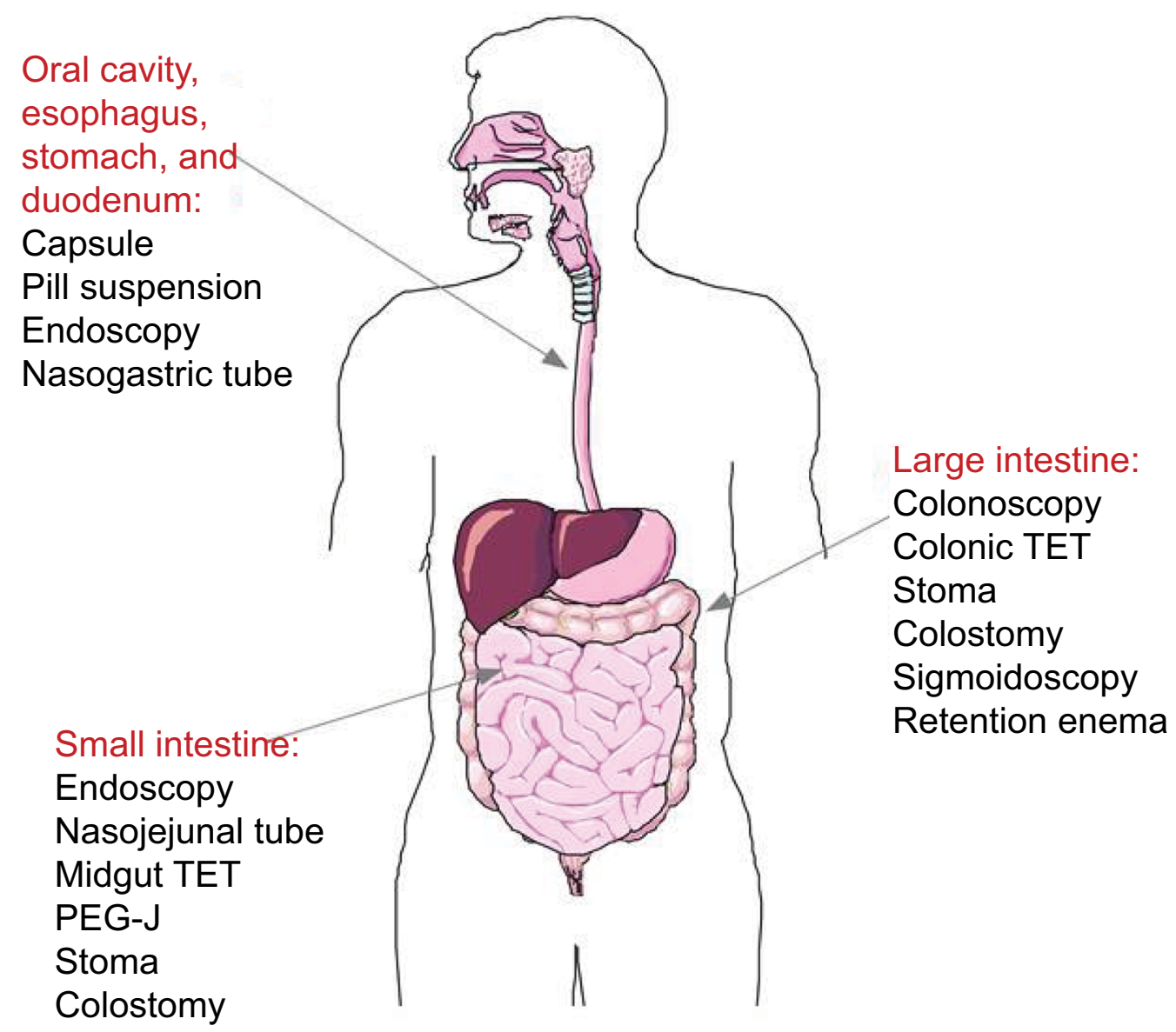

Figure I FMT: routes of administration.

Note: Images were created with SmartDraw software (https://www.smartdraw.com).

Abbreviations: FMT, fecal microbiota transplant; PEG-J, percutaneous endoscopic gastrojejunostomy; TET, transendoscopic enteral tubing. 
or stool test. The key issues addressed are about "infectious diseases," such as exposure to human immunodeficiency virus (HIV), hepatitis B virus or hepatitis C virus, syphilis, human T-lymphotropic virus I and II, malaria, trypanosomiasis, and tuberculosis; use of illegal drugs; risky sexual behavior; previous reception of organ transplant or blood products; recent ( $<6$ months) body tattoo, piercing, and acupuncture; recent ( $<6$ months) travel to tropical countries, countries at a high risk of transmissible diseases, or traveler's diarrhea; recent $(<6$ months) immunization with live attenuated virus; individual working with animals (increases the risk of zoonoses); "GI, metabolic, and neurological disorders," such as history of irritable bowel syndrome, IBD, chronic constipation, and other chronic GI disorders; history or high risk factor for GI cancer or polyposis; history of neurological/neurodegenerative disorders; history of psychiatric conditions; overweight and obesity (body mass index $>25$ ); and "drugs that can impair gut microbiota," such as recent exposure to antibiotics, immunotherapy, and chemotherapy as well as treatment with proton pump inhibitors. ${ }^{44}$ Donors who report significant exposure to one or more risk factors on their questionnaires are deemed to be unsuitable donors. In the second step, selected donors are scheduled for an in-person health assessment by the medical staff and are further checked for any recent onset of harmful events, by submitting a new form in which they briefly describe any changes to their health, diet, and bowel habits; travel to any tropical area; new sexual partners; and recent ingestion of substances that can be harmful for the recipient or that can affect the donor gut microbiota (antibiotics) since the previous screening. The above recommendations are supported by excellent safety data collected from several randomized clinical trials. ${ }^{18,21,45,46}$ In addition, stool specimens and blood are collected and analyzed for infectious diseases potentially transmittable to the recipient. Some tests are mandatory such as HIV; hepatitis A, B, and C; Salmonella, Shigella, and Campylobacter; ova and parasites; and Enterobacteriaceae, Enterococcus, Helicobacter pylori, and C. difficile, ${ }^{31}$ while others are optional according to geographical areas, clinical conditions of the recipients, and medical history of the donors. ${ }^{44}$ The questionnaire and test results are reviewed to determine the donors' suitability. Donors who test positive for potential transmissible pathogens are deemed and confidentially informed and referred for appropriate treatment. Standard donors must repeat the questionnaire and resubmit blood and stool specimens for testing every 3-6 months to confirm their suitability as donors. ${ }^{31}$ Of note, each donor has a different microbiota composition that makes difficult to obtain a homogenous FMT treatment, suggesting that an intrinsic characteristic of the stool donor may play a crucial role on the outcome of the FMT. In 2015, a study by Moayyedi et al observed that patients who received stool from the same donor (donor B) achieved remission more often than others, ${ }^{17,46}$ suggesting that the efficacy of the FMT is related to the donor stool "richness." Indeed, in the same year, a study by Grinspan and Kelly ${ }^{47}$ reported that the microbiota of donor B was enriched in members of the Lachnospiraceae family and Ruminococcus genus accounting for the greater efficacy of the FMT treatment. Same observations were described by Sokol in 2016. ${ }^{48}$ Moreover, another study revealed the importance of anti-inflammatory bacteria such as Faecalibacterium prausnitzii, whose levels are low in patients with Crohn's disease (CD). ${ }^{49,50}$ Therefore, the richness of the donor is an important parameter to take in consideration for a successful FMT. Another aspect to take into account is the donor-recipient microbiota compatibility. For instance, a donor strain belonging to a species already present in the recipient microbiota is more likely to establish in his/her gut, ${ }^{51}$ maximizing the FMT effect. Donor selection is a big challenge considering all the different aspects that can affect the outcome of FMT in IBD. Donor screening and consequently FMT activities can be facilitated by the development of stool banks. To do this, the Microbiome Health Research Institute (OpenBiome, Cambridge, MA, USA), a nonprofit organization dedicated to expanding safe access to FMT, has built an international public stool bank that allows to screen and process the donor stool in a standardized manner, at the same time offering a platform for investigating other microbiomeassociated diseases. ${ }^{52}$

\section{FMT in IBD}

The role of FMT for rCDI has been largely documented and demonstrated to have a cure rate of $90 \%$ in $>500$ reported cases to date. ${ }^{22,23}$ Considering the fact that microbiota composition is also profoundly modified in IBD, FMT is considered a promising approach for the treatment of IBD. Preliminary clinical reports of FMT in patients with ulcerative colitis (UC) and CD showed clinical remission that was maintained over long-term follow-up in many cases ${ }^{53,54}$ and in few other cases also reported endoscopic and histologic remission. ${ }^{55} \mathrm{~A}$ recent review and meta-analysis of nine studies that included 122 patients ( 79 with UC, $39 \mathrm{CD}$, and four with unclassified IBD) who received FMT found a remission rate of $36.2 \%{ }^{56}$ However, the remission was higher in younger patients (7-20 years old $)$ and in patients with CD $(64.1 \%$ and $60.5 \%$, respectively) compared with UC patients where only $22 \%$ achieved remission. Other small studies conducted in both adult and pediatric patients showed controversial results. Angelberger 
et al reported the results of a small study conducted in five patients with moderate to severe UC who received FMT via a nasojejunal tube. ${ }^{57}$ None of them showed remission after 12 weeks except for one patient who showed an improvement in the Mayo Score. In a different study involving four pediatric UC patients, Suskind et al did not observe any clinical improvement after a single administration of FMT via nasogastric tube. ${ }^{58}$ On the other hand, Kunde et al showed a high rate of success when they performed FMT via enema in nine pediatric UC patients once a day for 5 consecutive days. ${ }^{59}$ Indeed, seven patients showed remission after 1 week and six of them up to 1 month. Moreover, in a different study involving 15 adult patients with steroid-dependent UC who received FMT via upper colonoscopy, Cui et al reported clinical improvement in eight patients $(57 \%)$ with four of them maintaining long-term remission. ${ }^{32}$

Suskind et al were the first to report that FMT might be a therapeutic option in CD. ${ }^{60}$ Vaughn et al studied about increased intestinal microbial diversity following FMT in CD patients. In this study, eleven of 19 patients (58\%) showed improved clinical response. ${ }^{61}$ In another study by He et al, FMT efficacy and safety were evaluated in CD patients with inflammatory mass. A total of 25 patients were enrolled, and all the patients received initial FMT followed by FMT every 3 months; 17 of the 25 (68\%) patients achieved a clinical response, and 13 of the $25(52 \%)$ patients achieved clinical remission at 3 months. Sustained clinical remission was found in $12(48.0 \%)$, eight $(32.0 \%)$, and five $(22.7 \%)$ patients at 6,12 , and 18 months, respectively. ${ }^{62}$ A study of 30 patients by Cui et al on FMT through midgut for refractory $\mathrm{CD}$ showed that the clinical improvement and remission rates based on clinical activity at the first month were $86.7 \%$ (26 of 30 ) and $76.7 \%$ (23 of 30$){ }^{63}$

Unfortunately, these studies lack uniformity in treatment protocols and route of administration and did not include control (placebo) groups; therefore, it is complicated to make a solid conclusion about the safety and efficacy of FMT in IBD. Recently, two randomized placebo control trials evaluating the efficacy of FMT in IBD were published. In a study by Moayyedi et al, 75 patients with UC were randomized to receive FMT or placebo via enema once a week for 6 weeks. ${ }^{46}$ The results showed that the treated group achieved higher remission compared with the placebo ( $24 \%$ vs $9 \%)$. The second trial enrolled 50 patients with mild to severe UC and randomized to receive stool from a healthy donor or autologous fecal microbiota via nasoduodenal delivery at weeks 0 and $3 .{ }^{45}$ There was no statistically significant difference in clinical or endoscopic remission between the two groups.
Altogether, the results of FMT in IBD may look disappointing, especially if compared with the impressive results obtained in patients with rCDI (about 90\% remission). However, these data highlight the fact that IBD is not a pure microbiota-driven disease such as CDI, but it is far more complex, suggesting that results obtained in CDI trials cannot be directly transposed to IBD. Furthermore, the studies of FMT in IBD have involved only a few patients so far and are quite inconsistent in many aspects, such as the age (pediatric vs adult patients), the control groups, dose and preparation of donor feces, delivery method, and frequency of FMT. Therefore, more randomized controlled placebo studies are needed to clarify and optimize the role of FMT in IBD.

\section{Adverse effects of FMT}

Potential side effects of FMT can be categorized into shortterm and long-term. While short-term events can also be distinguished between side effects related to the delivery method or to FMT itself, very little information exists regarding long-term events considering the lack of lengthy prospective trials to assess its safety.

\section{Short-term side effects}

Regardless of the delivery method, the common side effects following FMT include a mild fever and mild GI symptoms (abdominal discomfort, flatulence, diarrhea, constipation, and vomiting) that usually resolve within few weeks. ${ }^{18,57,64}$ The route of administration seems to affect the side effects profile. For example, high fever and rise of the C-reactive protein have been described via the nasojejunal route ${ }^{65}$ and rarely have been reported for aspiration pneumonia, ${ }^{66}$ while perforation, bleeding, and effects related to anesthesia have been described for the colonoscopy. ${ }^{23}$ Transmission of enteric pathogens through fecal donor material appears to be rare considering the screening procedure that donors undergo. However, two cases of intestinal infection (norovirus) at 2 and 12 days post-FMT were reported ${ }^{67}$ Mortality has been reported in the literature. ${ }^{17,68}$ One case described an aspiration event related to the delivery method of FMT, while in the second case, the patient died 13 days after FMT secondary to progressive pneumonia, for which the patient was treated with antibiotics before and after FMT. However, the latter can be unrelated to FMT, but rather associated with patient's comorbidity.

\section{Long-term side effects}

The major concern about the safety of FMT in IBD is the assessment of long-term side effects. However, due to the 
lack of long prospective studies, there is not much information collected about long-term side effects, and many are speculative. A great theoretical risk may be the induction of chronic diseases based on the alteration of gut microbiota, which includes obesity, diabetes, atherosclerosis, and colon cancer. Significant gain of weight was reported in a woman who received FMT from an overweight donor. The recipient was also overweight at the time of FMT; thus, the data must be interpreted with caution. ${ }^{69}$ However, studies in rodents have corroborated the abovementioned hypothesis. Some groups have reported transferring colitis from different knockout models to wild-type mice. ${ }^{70,71}$ The transplantation of human microbiota from obese subjects to rodents leads to obesity in rodents, ${ }^{72}$ and FMT from lean donors increases insulin sensitivity in obese individuals with metabolic syndrome and thus legitimates the concern about the long-term risks of FMT. Finally, long-term follow-up combined with the analysis of screened donors and recipient specimens will be crucial to assess the safety and future adverse events.

\section{Contraindications to FMT}

FMT is often perceived as "natural" remedy by many patients and physicians, However, considering the fact that the transfer of complex microbiota can modify the host phenotype with unknown long-term effects, it is preferable to exclude certain categories of patients in which the delivery of FMT may worsen their condition, or it may even be fatal. For example, patients with severe bowel disease cannot undergo colonoscopy, while those with severe immunosuppression and decompensated liver cirrhosis are excluded considering the potential risk of enteric microbe transmission from donor's stool. However, recently, Kelly et al published a retrospective study of immunocompromised patients who received FMT to treat $\mathrm{rCDI}$ in which they show that there were no infectious complications following FMT in these potentially "at-risk" patients. ${ }^{23}$

\section{Discussion}

IBD is a chronic intestinal disorder, causes of which are not fully elucidated yet. Despite the availability of different therapeutic options, treatment dissatisfaction is still high, implying a reduced life quality of the patients and increased social, sanitary, and economic burden worldwide. Therefore, the identification of new players involved in the physiopathology of IBD will improve and expand the therapeutic armamentarium. ${ }^{73}$

As early as the 1900s, physicians recognized that bacteria might promote development and maintenance of symptoms in colitis. ${ }^{74}$ In the last decade, thanks to advanced molecular techniques, profound differences in the composition and functions of the microbiota have been demonstrated in patients with IBD compared with healthy subjects, ${ }^{75-77}$ recognizing a role of the gut microbiome in the etiology and pathogenesis of IBD. The high therapeutic efficacy of FMT in the treatment of rCDI is impressive, and although the microbial basis of IBD is far more complex and variable than that of rCDI, microbiome-based therapies are an important area of investigation for these chronic diseases. FMT is the first way to directly alter the intestinal microbiome; therefore, it is considered a promising therapy in IBD. However, standardized controlled studies are necessary to define which patient is eligible, frequency and optimal timing in which FMT should be proposed (at the early or late point of the disease course), and the optimal donor for each patient. Robust safety data of FMT are still missing, and long-term clinical follow-up will be crucial to address this issue. In addition, animal and human data showed that donor microbiota could induce certain chronic disease that is gut-driven. ${ }^{69-72}$ Thus, more information is necessary on the modification of host microbiota composition and future research on metabolic pathways and microbial genes in the GI tract, and their effects on protein expression will shed light on the role of the gut microbiota in these chronic conditions. Moreover, it will be interesting to identify active components of the gut microbiota that could be isolated and used as therapeutic agents in IBD. The rationale for that comes from the studies of Sokol et al, which identified the protective role of the F. prausnitzii in the intestinal inflammatory process. ${ }^{49}$ Recently, the anti-inflammatory molecule produced by this commensal bacterium has been identified, ${ }^{78,79}$ suggesting that, in the near future, "artificial" microbiota can be generated and used to counterbalance the dysbiosis in a more standardized and controlled way compared with FMT. Finally, it is well accepted that IBD pathogenesis is related to an aberrant cross talk between the host immune system and gut microbiota; ${ }^{3}$ however, thus far, the only approach envisaged was to inhibit the overactivated immune system. With our current understanding of the microbiological basis for IBD, it should be taken into account both players, and probably it should target them simultaneously to achieve optimal results.

\section{Author contributions}

TS and PR contributed to conception and design. TS, PR, $\mathrm{AO}$, and $\mathrm{VG}$ contributed to analysis and interpretation. PR, TS, AO, and VG drafted the article. TS, PR, AO, and VG critically revised the article and approved the final version of the article. All authors contributed toward data analysis, drafting and revising the paper and agree to be accountable for all aspects of the work. 


\section{Disclosure}

The authors report no conflicts of interest in this work.

\section{References}

1. Lovell RM, Ford AC. Global prevalence of and risk factors for irritable bowel syndrome: a meta-analysis. Clin Gastroenterol Hepatol. 2012;10(7):712.e4-721.e4.

2. Barbara G, Feinle-Bisset C, Ghoshal UC, et al. The intestinal microenvironment and functional gastrointestinal disorders. Gastroenterology. 2016;150(6):1305-1318

3. Khor B, Gardet A, Xavier RJ. Genetics and pathogenesis of inflammatory bowel disease. Nature. 2011;474(7351):307-317.

4. Gazouli M, Wouters MM, Kapur-Pojskić L, et al. Lessons learned resolving the enigma of genetic factors in IBS. Nat Rev Gastroenterol Hepatol. 2016;13(2):77-87.

5. Murgas Torrazza R, Neu J. The developing intestinal microbiome and its relationship to health and disease in the neonate. $J$ Perinatol. 2011;31(Suppl 1):S29-S34.

6. Round JL, Lee SM, Li J, et al. The Toll-like receptor 2 pathway establishes colonization by a commensal of the human microbiota. Science. 2011;332(6032):974-977.

7. Ivanov II, Atarashi K, Manel N, et al. Induction of intestinal Th17 cells by segmented filamentous bacteria. Cell. 2009;139(3):485-498.

8. Candela M, Perna F, Carnevali P, et al. Interaction of probiotic Lactobacillus and Bifidobacterium strains with human intestinal epithelial cells: adhesion properties, competition against enteropathogens and modulation of IL-8 production. Int J Food Microbiol. 2008;125(3):286-292.

9. Matsumoto M, Kibe R, Ooga T, et al. Cerebral low-molecular metabolites influenced by intestinal microbiota: a pilot study. Front Syst Neurosci. 2013;7:9.

10. Cryan JF, Dinan TG. Mind-altering microorganisms: the impact of the gut microbiota on brain and behaviour. Nat Rev Neurosci. 2012;13(10):701-712.

11. Gevers D, Kugathasan S, Denson LA, et al. The treatment-naive microbiome in new-onset Crohn's disease. Cell Host Microbe. 2014;15(3):382-392.

12. Morgan XC, Tickle TL, Sokol H, et al. Dysfunction of the intestinal microbiome in inflammatory bowel disease and treatment. Genome Biol. 2012;13(9):R79.

13. Sokol H, Seksik P. The intestinal microbiota in inflammatory bowel diseases: time to connect with the host. Curr Opin Gastroenterol. 2010;26(4):327-331.

14. Henson MA, Phalak P. Microbiota dysbiosis in inflammatory bowel diseases: in silico investigation of the oxygen hypothesis. BMC Syst Biol. 2017;11(1):145.

15. Kim D, Zeng MY, Núñez G. The interplay between host immune cells and gut microbiota in chronic inflammatory diseases. Exp Mol Med. 2017;49(5):e339.

16. Shen ZH, Zhu CX, Quan YS, et al. Relationship between intestinal microbiota and ulcerative colitis: mechanisms and clinical application of probiotics and fecal microbiota transplantation. World J Gastroenterol. 2018;24(1):5-14.

17. Kelly CR, Kahn S, Kashyap P, et al. Update on fecal microbiota transplantation 2015: indications, methodologies, mechanisms, and outlook Gastroenterology. 2015;149(1):223-237.

18. van Nood E, Vrieze A, Nieuwdorp M, et al. Duodenal infusion of donor feces for recurrent Clostridium difficile. $N$ Engl $J$ Med 2013;368(5):407-415.

19. Leffler DA, Lamont JT. Clostridium difficile infection. $N$ Engl J Med. 2015;372(16):1539-1548.

20. Austin M, Mellow M, Tierney WM. Fecal microbiota transplantation in the treatment of Clostridium difficile infections. Am J Med. 2014;127(6):479-483.

21. Cammarota G, Masucci L, Ianiro G, et al. Randomised clinical trial: faecal microbiota transplantation by colonoscopy vs. vancomycin for the treatment of recurrent Clostridium difficile infection. Aliment Pharmacol Ther. 2015;41(9):835-843.
22. Kassam Z, Lee CH, Yuan Y, Hunt RH. Fecal microbiota transplantation for Clostridium difficile infection: systematic review and meta-analysis. Am J Gastroenterol. 2013;108(4):500-508.

23. Kelly CR, Ihunnah C, Fischer M, et al. Fecal microbiota transplant for treatment of Clostridium difficile infection in immunocompromised patients. Am J Gastroenterol. 2014;109(7):1065-1071.

24. Moore T, Rodriguez A, Bakken JS. Fecal microbiota transplantation: a practical update for the infectious disease specialist. Clin Infect Dis. 2014;58(4):541-545.

25. Zhang F, Cui B, He X, et al. Microbiota transplantation: concept, methodology and strategy for its modernization. Protein Cell. 2018;9(5):462-473.

26. Gough E, Shaikh H, Manges AR. Systematic review of intestinal microbiota transplantation (fecal bacteriotherapy) for recurrent Clostridium difficile infection. Clin Infect Dis. 2011;53(10):994-1002.

27. Costello SP, Tucker EC, La Brooy J, Schoeman MN, Andrews JM Establishing a fecal microbiota transplant service for the treatment of Clostridium difficile infection. Clin Infect Dis. 2016;62(7):908-914.

28. Mattila E, Uusitalo-Seppälä R, Wuorela M, et al. Fecal transplantation, through colonoscopy, is effective therapy for recurrent Clostridium difficile infection. Gastroenterology. 2012;142(3):490-496.

29. Satokari R, Mattila E, Kainulainen V, Arkkila PE. Simple faecal preparation and efficacy of frozen inoculum in faecal microbiota transplantation for recurrent Clostridium difficile infection - an observational cohort study. Aliment Pharmacol Ther. 2015;41(1):46-53.

30. Liao CH, Shollenberger LM. Survivability and long-term preservation of bacteria in water and in phosphate-buffered saline. Lett Appl Microbiol. 2003;37(1):45-50.

31. Tauxe WM, Dhere T, Ward A, Racsa LD, Varkey JB, Kraft CS. Fecal microbiota transplant protocol for clostridium difficile infection. $L a b$ Med. 2015;46(1):e19-e23.

32. Cui B, Li P, Xu L, et al. Step-up fecal microbiota transplantation strategy: a pilot study for steroid-dependent ulcerative colitis. JTransl Med. 2015;13:298.

33. Youngster I, Russell GH, Pindar C, Ziv-Baran T, Sauk J, Hohmann EL. Oral, capsulized, frozen fecal microbiota transplantation for relapsing Clostridium difficile infection. JAMA. 2014;312(17):1772-1778.

34. Hamilton MJ, Weingarden AR, Sadowsky MJ, Khoruts A. Standardized frozen preparation for transplantation of fecal microbiota for recurrent Clostridium difficile infection. Am J Gastroenterol. 2012;107(5):761-767.

35. Fuller BJ. Cryoprotectants: the essential antifreezes to protect life in the frozen state. Cryo Letters. 2004;25(6):375-388.

36. Bahl MI, Bergström A, Licht TR. Freezing fecal samples prior to DNA extraction affects the Firmicutes to Bacteroidetes ratio determined by downstream quantitative PCR analysis. FEMS Microbiol Lett. 2012;329(2):193-197.

37. Kelly CR, Khoruts A, Staley C, et al. Effect of fecal microbiota transplantation on recurrence in multiply recurrent Clostridium difficile infection: a randomized trial. Ann Intern Med. 2016;165(9):609-616.

38. Aas J, Gessert CE, Bakken JS. Recurrent Clostridium difficile colitis: case series involving 18 patients treated with donor stool administered via a nasogastric tube. Clin Infect Dis. 2003;36(5):580-585.

39. Hirsch BE, Saraiya N, Poeth K, Schwartz RM, Epstein ME, Honig G. Effectiveness of fecal-derived microbiota transfer using orally administered capsules for recurrent Clostridium difficile infection. BMC Infect Dis. 2015; 15:191.

40. Peng Z, Xiang J, He Z, et al. Colonic transendoscopic enteral tubing: a novel way of transplanting fecal microbiota. Endosc Int Open. 2016;4(6):E610-E613.

41. Ni X, Fan S, Zhang Y, et al. Coordinated hospital-home fecal microbiota transplantation via percutaneous endoscopic cecostomy for recurrent steroid-dependent ulcerative colitis. Gut Liver. 2016;10(6):975-980.

42. David LA, Maurice CF, Carmody RN, et al. Diet rapidly and reproducibly alters the human gut microbiome. Nature. 2014;505(7484):559-563.

43. Rawls JF, Mahowald MA, Ley RE, Gordon JI. Reciprocal gut microbiota transplants from zebrafish and mice to germ-free recipients reveal host habitat selection. Cell. 2006;127(2):423-433. 
44. Cammarota G, Ianiro G, Tilg H, et al. European consensus conference on faecal microbiota transplantation in clinical practice. Gut. 2017;66(4):569-580.

45. Rossen NG, Fuentes S, van der Spek MJ, et al. Findings from a randomized controlled trial of fecal transplantation for patients with ulcerative colitis. Gastroenterology. 2015;149(1):110.e4-118.e4.

46. Moayyedi P, Surette MG, Kim PT, et al. Fecal microbiota transplantation induces remission in patients with active ulcerative colitis in a randomized controlled trial. Gastroenterology. 2015;149(1):102.e6-109.e6.

47. Grinspan AM, Kelly CR. Fecal microbiota transplantation for ulcerative colitis: not just yet. Gastroenterology. 2015;149(1):15-18.

48. Sokol H. Toward rational donor selection in faecal microbiota transplantation for IBD. J Crohns Colitis. 2016;10(4):375-376.

49. Sokol H, Pigneur B, Watterlot L, et al. Faecalibacterium prausnitzii is an anti-inflammatory commensal bacterium identified by gut microbiota analysis of Crohn disease patients. Proc Natl Acad Sci U S A. 2008;105(43):16731-16736.

50. Sokol H, Seksik P, Furet JP, et al. Low counts of Faecalibacterium prausnitzii in colitis microbiota. Inflamm Bowel Dis. 2009;15(8):1183-1189.

51. Li SS, Zhu A, Benes V, et al. Durable coexistence of donor and recipient strains after fecal microbiota transplantation. Science. 2016;352(6285):586-589.

52. Kazerouni A, Burgess J, Burns LJ, Wein LM. Optimal screening and donor management in a public stool bank. Microbiome. 2015;3:75.

53. Bennet JD, Brinkman M. Treatment of ulcerative colitis by implantation of normal colonic flora. Lancet. 1989;1(8630):164.

54. Borody TJ, George L, Andrews P, et al. Bowel-flora alteration: a potential cure for inflammatory bowel disease and irritable bowel syndrome? Med J Aust. 1989;150(10):604.

55. Borody TJ, Warren EF, Leis S, Surace R, Ashman O. Treatment of ulcerative colitis using fecal bacteriotherapy. J Clin Gastroenterol. 2003;37(1):42-47.

56. Colman RJ, Rubin DT. Fecal microbiota transplantation as therapy for inflammatory bowel disease: a systematic review and meta-analysis. $J$ Crohns Colitis. 2014;8(12):1569-1581.

57. Angelberger S, Reinisch W, Makristathis A, et al. Temporal bacterial community dynamics vary among ulcerative colitis patients after fecal microbiota transplantation. Am J Gastroenterol. 2013;108(10):1620-1630

58. Suskind DL, Singh N, Nielson H, Wahbeh G. Fecal microbial transplant via nasogastric tube for active pediatric ulcerative colitis. $J$ Pediatr Gastroenterol Nutr. 2015;60(1):27-29.

59. Kunde S, Pham A, Bonczyk S, et al. Safety, tolerability, and clinical response after fecal transplantation in children and young adults with ulcerative colitis. J Pediatr Gastroenterol Nutr. 2013;56(6):597-601.

60. Suskind DL, Brittnacher MJ, Wahbeh G, et al. Fecal microbial transplant effect on clinical outcomes and fecal microbiome in active Crohn's disease. Inflamm Bowel Dis. 2015;21(3):556-563.

61. Vaughn BP, Vatanen T, Allegretti JR, et al. Increased intestinal microbial diversity following fecal microbiota transplant for active Crohn's disease. Inflamm Bowel Dis. 2016;22(9):2182-2190.
62. He Z, Li P, Zhu J, et al. Multiple fresh fecal microbiota transplants induces and maintains clinical remission in Crohn's disease complicated with inflammatory mass. Sci Rep. 2017;7(1):4753.

63. Cui B, Feng Q, Wang H, et al. Fecal microbiota transplantation through mid-gut for refractory Crohn's disease: safety, feasibility, and efficacy trial results. J Gastroenterol Hepatol. 2015;30(1):51-58.

64. Kump PK, Gröchenig HP, Lackner S, et al. Alteration of intestinal dysbiosis by fecal microbiota transplantation does not induce remission in patients with chronic active ulcerative colitis. Inflamm Bowel Dis. 2013;19(10):2155-2165.

65. Vermeire S, Joossens M, Verbeke K, et al. Donor species richness determines faecal microbiota transplantation success in inflammatory bowel disease. J Crohns Colitis. 2016;10(4):387-394.

66. Baxter M, Ahmad T, Colville A, Sheridan R. Fatal aspiration pneumonia as a complication of fecal microbiota transplant. Clin Infect Dis. 2015;61(1):136-137.

67. Schwartz M, Gluck M, Koon S. Norovirus gastroenteritis after fecal microbiota transplantation for treatment of Clostridium difficile infection despite asymptomatic donors and lack of sick contacts. $\mathrm{Am} \mathrm{J}$ Gastroenterol. 2013;108(8):1367.

68. Sha S, Liang J, Chen M, et al. Systematic review: faecal microbiota transplantation therapy for digestive and nondigestive disorders in adults and children. Aliment Pharmacol Ther. 2014;39(10):1003-1032.

69. Alang N, Kelly CR. Weight gain after fecal microbiota transplantation. Open Forum Infect Dis. 2015;2(1):ofv004.

70. Garrett WS, Lord GM, Punit S, et al. Communicable ulcerative colitis induced by T-bet deficiency in the innate immune system. Cell. 2007;131(1):33-45.

71. Elinav E, Strowig T, Kau AL, et al. NLRP6 inflammasome regulates colonic microbial ecology and risk for colitis. Cell. 2011;145(5):745-757.

72. Ridaura VK, Faith JJ, Rey FE, et al. Gut microbiota from twins discordant for obesity modulate metabolism in mice. Science. 2013;341(6150):1241214

73. Rawla P, Sunkara T, Raj JP. Role of biologics and biosimilars in inflammatory bowel disease: current trends and future perspectives. J Inflamm Res. 2018;11:215-226.

74. Wallis FC. The surgery of colitis. Br Med J. 1909;1(2505):10-13.

75. Nagalingam NA, Lynch SV. Role of the microbiota in inflammatory bowel diseases. Inflamm Bowel Dis. 2012;18(5):968-984.

76. Manichanh C, Borruel N, Casellas F, Guarner F. The gut microbiota in IBD. Nat Rev Gastroenterol Hepatol. 2012;9(10):599-608.

77. Frank DN, St Amand AL, Feldman RA, Boedeker EC, Harpaz N, Pace NR. Molecular-phylogenetic characterization of microbial community imbalances in human inflammatory bowel diseases. Proc Natl Acad Sci US A. 2007;104(34):13780-13785.

78. Miquel S, Leclerc M, Martin R, et al. Identification of metabolic signatures linked to anti-inflammatory effects of Faecalibacterium prausnitzii. MBio. 2015;6(2):pii.e00300-15.

79. Quévrain E, Maubert MA, Michon C, et al. Identification of an antiinflammatory protein from Faecalibacterium prausnitzii, a commensal bacterium deficient in Crohn's disease. Gut. 2016;65(3):415-425.
Journal of Inflammation Research

\section{Publish your work in this journal}

The Journal of Inflammation Research is an international, peer-reviewed open access journal that welcomes laboratory and clinical findings on the molecular basis, cell biology and pharmacology of inflammation including original research, reviews, symposium reports, hypothesis formation and commentaries on: acute/chronic inflammation; mediators of
Dovepress

inflammation; cellular processes; molecular mechanisms; pharmacology and novel anti-inflammatory drugs; clinical conditions involving inflammation. The manuscript management system is completely online and includes a very quick and fair peer-review system. Visit http://www.dove press.com/testimonials.php to read real quotes from published authors. 